CLINICAL STUDY

\title{
A clinical study on the short-term effect of berberine in comparison to metformin on the metabolic characteristics of women with polycystic ovary syndrome
}

\author{
Wei Wei ${ }^{1, *}$, Hongmin Zhao ${ }^{1, *}$, Aili Wang ${ }^{1}$, Ming Sui ${ }^{1}$, Kun Liang ${ }^{1}$, Haiyun Deng ${ }^{1}$, Yukun Ma $^{1}$, Yajuan Zhang ${ }^{1}$, \\ Hongxiu Zhang ${ }^{1}$ and Yuanyuan Guan ${ }^{1,2}$ \\ ${ }^{1}$ Department of Gynecology and Obstetrics, Clinical Center of Reproductive Medicine, the First Affiliated Hospital of Harbin Medical University, Harbin \\ Medical University, 23 Youzheng Street, Nangang District, Harbin, Heilongjiang 150001, People's Republic of China and ${ }^{2}$ Clinical Center of Reproductive \\ Medicine, Peking Union Medical College Hospital, Beijin 100001, People's Republic of China
}

(Correspondence should be addressed to Y Guan at Department of Gynecology and Obstetrics, Clinical Center of Reproductive Medicine, the First Affiliated Hospital of Harbin Medical University, Harbin Medical University; Email: guanyuanyuan63@yahoo.cn)

*(W Wei and H Zhao contributed equally to this work)

\begin{abstract}
Objective: Polycystic ovary syndrome (PCOS) is a frequent reproductive and metabolic disorder associated with insulin resistance (IR). Berberine (BBR) is an isoquinoline derivative alkaloid extracted from Chinese medicinal herbs that has been used as an insulin sensitizer. BBR may have a potential therapeutic value for PCOS. The aim of this study was to evaluate the effects of BBR in comparison to metformin (MET) on the metabolic features of women with PCOS.

Design and methods: Eighty-nine subjects with PCOS and IR subjects were randomized into one of three treatment groups: BBR + compound cyproterone acetate (CPA; $n=31)$, MET + CPA $(n=30)$, and placebo + CPA $(n=28)$ for 3 months. Clinical characteristics of the women and metabolic and hormonal parameters were assessed before and after the period of treatment.

Results: Treatment with BBR in comparison to MET showed decrease in waist circumference and waistto-hip ratio (WHR; $P<0.01$ ), total cholesterol (TC), triglycerides (TG), and low-density lipoprotein cholesterol (LDLC; $P<0.05$ ) as well as increase in high-density lipoprotein cholesterol (HDLC) and sex hormone-binding globulin (SHBG; $P<0.05)$. Similarly, treatment with BBR in comparison to placebo showed decrease in WHR, fasting plasma glucose, fasting insulin, homeostasis model assessment for IR, area under the curve of insulin, TC, LDLC, and TG $(P<0.05)$ as well as increase in HDLC and SHBG $(P<0.01)$.

Conclusions: Intake of BBR improved some of the metabolic and hormonal derangements in a group of treated Chinese women with PCOS. Main effects could be related to the changes in body composition in obesity and dyslipidemia. Further controlled studies are needed for the assessment of the potential favorable metabolic effects of BBR in women with PCOS.
\end{abstract}

European Journal of Endocrinology 166 99-105

\section{Introduction}

Polycystic ovary syndrome (PCOS) is a common endocrine and metabolic disorder afflicting $3-8 \%$ of women of reproductive age. PCOS is characterized by chronic anovulation, hyperandrogenism, and/or the presence of polycystic ovary morphology (1). A wide variety of risk factors have been investigated in relation to PCOS, including obesity, glucose intolerance, and dyslipidemia, which lead to a significantly increased risk for long-term type 2 diabetes mellitus (T2DM) and cardiovascular disease (2-5). Insulin resistance (IR) is known to play a critical role in the pathophysiology of PCOS $(6,7)$. The administration of insulin sensitizer metformin (MET) is recognized as a successful treatment for many metabolic and reproductive dysregulations characteristic of women with PCOS (8-13).

Berberine (BBR, $\left[\mathrm{C}_{20} \mathrm{H}_{18} \mathrm{NO}_{4}\right]$ ) is a type of isoquinoline derivative alkaloid extracted from Chinese medicinal herbs, such as Coptidis Rhizoma (Huanglian), Cortex Phellodendri (Huangbai), and Hydrastis Canadensis (goldenseal). In China, BBR is a non-prescription oral drug for the treatment of gastrointestinal infections and diabetes. BBR possesses a wide range of biochemical and pharmacological activities, including anti-obesity and anti-dyslipidemia (14-16). A large body of evidence has demonstrated that BBR is an effective insulin sensitizer and has a comparable activity to MET in reducing IR $(15,17-19)$. However, before we can draw conclusions regarding the pharmacological 
properties of BBR and their applicability to the pathophysiological changes observed in PCOS, trials examining the therapeutic value of BBR in patients with PCOS are required.

This study aimed to assess whether use of BBR, considered to be an insulin sensing agent, is effective in the treatment of endocrine characteristics of PCOS and to compare these effects with MET therapy. The outcome measures in this study included change in anthropometric measures and hormonal and metabolic indices in a group of insulin-resistant Chinese women with PCOS.

\section{Subjects and methods}

\section{Subjects}

A total of 100 subjects with PCOS and insulin resistance (IR) were included in this study. They were recruited from the Clinical Centre of Reproductive Medicine of the First Affiliated Hospital of Harbin Medical University (Harbin, China). The diagnostic criteria for PCOS followed the 2003 Rotterdam ESHRE/ASRM criteria. Oligomenorrhea (interval between menstrual periods $\geq 35$ days) or amenorrhea (absence of vaginal bleeding for at least 6 months) (20) and clinical (a FerrimanGallwey score $\geq 6$ ) (21) and/or biochemical hyperandrogenism (total testosterone (TT) $\geq 58 \mathrm{ng} / \mathrm{dl}(2 \mathrm{nmol} / \mathrm{l})$ ) were used to assess PCOS. Normal range of TT in healthy Chinese women is no more than $2 \mathrm{nmol} / \mathrm{l}$ (22). The phenotype of polycystic ovaries was detected by vaginal ultrasound examination presenting 12 follicles or more in one or both ovaries and/or increased ovarian volume $(>10 \mathrm{ml})$ (23). All subjects fulfilled at least two of the three diagnostic criteria. IR was assessed by homeostasis model assessment for IR (HOMA-IR) $\geq 3.8$ (24) or fasting glucose insulin ratio (FGIR) $\leq 4.5$ (25).

Exclusion conditions included the following systemic and endocrine disorders: late-onset congenital adrenal hyperplasia, Cushing's syndrome, thyroid dysfunction, hyperprolactinemia, diabetes mellitus, coronary artery disease, and spontaneous abortion. Furthermore, subjects accepting treatment with medications known to alter insulin hemodynamics, ovulation induction, anti-obesity, or oral contraceptives (OCs) within 3 months were excluded from the study. All subjects were nonsmokers, and none reported chronic alcohol consumption.

The study was conducted according to the Helsinki Declaration on human experimentation. The study protocol was approved by the ethics committee of the First Affiliated Hospital of Harbin Medical University. The registration number of the trial is 200907. The purpose, procedure, and potential risks of the study were carefully explained to each subject, and written consent was obtained before beginning the study.

\section{Methods}

All subjects received lifestyle modification and antiandrogen compound cyproterone acetate (CPA) in the form of combined pill with ethinyl estradiol, randomly assigned to the BBR, MET, or placebo groups for a 3-month treatment course. Randomization was based on a computer-generated code in blocks of six. A copy of the code was stored in a sealed envelope by personnel not involved in the trial.

All subjects received advice from a nutritionist on nutrition and exercise. They were instructed to limit fat and carbohydrate intake and improve dietary behavior without the application of a calorie-restricted diet program. Exercise was also recommended to include $30 \mathrm{~min} /$ day of moderate to intense activity and was not monitored. CPA (Diane 35, Bayer Schering Pharma) containing $35 \mu \mathrm{g}$ ethinyl estradiol and $2.0 \mathrm{mg}$ cyproterone acetate were also taken in a cyclic fashion. BBR (BBR hydrochloride, Northeast General Pharmaceutical Factory, Harbin, China) was administered at a dosage of $3 \times 500 \mathrm{mg}$ daily, MET (Bristol-Myers Squibb Company, Shanghai, China) was administered at a dosage of $3 \times$ $500 \mathrm{mg}$ daily, except for the first week of treatment when $500 \mathrm{mg}$ was given only twice a day to reduce the incidence and severity of gastrointestinal side effects. Placebo (provided by pharmaceutical preparation section) was administered as one tablet twice a day.

\section{Clinical and endocrine examination}

Clinical assessments included anthropometric measurements (height $(\mathrm{m})$, weight $(\mathrm{kg})$, body mass index (BMI), and waist-to-hip ratio (WHR)). BMI was calculated as weight divided by height squared. WHR was defined as the ratio between the circumference of waist and hip. Blood pressure was measured in the seated position in the right arm after a $30 \mathrm{~min}$ rest period and averaged over three measurements.

In the baseline study, vaginal bleeding in the absence of spontaneous bleeding was induced by progesterone withdrawal. After a 12 h overnight fast, a venous blood sample was obtained in the morning between 0800 and $0900 \mathrm{~h}$ during the early follicular phase (days 2-3). In all subjects, circulating concentrations of the reproductive hormones TT and sex hormone-binding globulin (SHBG) were measured. The free androgen index $(\mathrm{FAI})=$ (testosterone $(\mathrm{nmol} / \mathrm{l}) / \mathrm{SHBG}(\mathrm{nmol} / \mathrm{l})) \times 100(26)$ was also calculated. Other parameters included fasting plasma glucose (FPG) and insulin (FIN), total cholesterol (TC), triglycerides (TG), high-density lipoprotein cholesterol (HDLC), and low-density lipoprotein cholesterol (LDLC). Immediately after obtaining the basal blood sample, a $2 \mathrm{~h}, 75 \mathrm{~g}$ oral glucose tolerance test (OGTT) was performed, and blood samples were obtained after 30,60 , and $120 \mathrm{~min}$ to measure serum glucose and insulin concentrations. The degree of IR was estimated by a number of different methods, including calculated 
FGIR, HOMA-IR, and area under the insulin curve $\left(\mathrm{AUC}_{\mathrm{INS}}\right)$. Impaired glucose tolerance (IGT) was defined as fasting glucose $>5.6 \mathrm{mmol} / \mathrm{l}$ and/or $2-\mathrm{h}$ glucose $>7.8 \mathrm{mmol} / \mathrm{l}(27)$.

$\mathrm{FGIR}=\mathrm{FPG}(\mathrm{mg} / \mathrm{dl}) / \mathrm{FIN}(\mathrm{mIU} / \mathrm{ml})(24)$.

HOMA-IR $=$ FIN $(\mathrm{mIU} / \mathrm{ml}) \times$ FPG $(\mathrm{mmol} / \mathrm{l}) / 22.5$ (23).

$\mathrm{AUC}_{\mathrm{INS}}=$ insulin $0^{\prime} \times 0.25+$ insulin $30^{\prime} \times 0.5+$ insulin $60^{\prime} \times 0.75+$ insulin $120^{\prime} \times 0.5(28)$.

After 3 months of treatment, each subject underwent the same procedures as described above.

\section{Assays}

Serum concentrations of TT, SHBG, and insulin were measured using the electro-chemiluminescent immunoassay method by auto-analyzer (Abbott I2000). Plasma glucose, TC, TG, HDLC, and LDLC levels were determined using the electro-chemiluminescent immunoassay method by auto-analyzer (Abbott V51).

\section{Statistical analysis}

SPSS 13.0 (SPSS Inc., Chicago, IL, USA) was used for the data analysis. A repeated measures ANOVA was used to estimate the statistical difference between treatment groups, including pre- and post-treatment and within one group between pre- and post-treatment. Logarithmic transformations of FGIR, HOMA-IR, and $\mathrm{AUC}_{\mathrm{INS}}$ were applied before ANOVA to ensure homogeneity of variances. Data are presented as mean \pm s.D. with a value of $P<0.05$ considered as statistically significant.

\section{Results}

All subjects were reproductive-aged Chinese females living in the Heilongjiang Province. Complete data from 89 of the 100 subjects were analyzed. One subject from the BBR group, two from the MET group, and two from the placebo group were lost to follow-up after randomization without any visits. Two subjects from the BBR group and one from the MET group left for personal reasons. Two subjects from the MET group and one from the placebo group left because of travel difficulties. The final number of subjects in the three groups were similar (BBR 30, MET 31, and placebo 28). Nine subjects who received MET complained of transient abdominal discomfort (nausea, vomiting, mild diarrhea, and flatulence) and three subjects who received BBR complained of a bitter taste in their mouth.

\section{Clinical characteristics of the studied subjects}

At baseline, there were no significant differences in any parameter among the groups (Table 1). After 3 months of treatment, body weight and BMI decreased significantly in all three groups; however, compared to placebo, no significant differences in body weight and BMI were observed in the BBR $(P=0.928, P=0.161)$ and the MET groups $(P=0.146, P=0.975)$. BBR and MET had no additive effect on decreasing weight and BMI. Waist circumference (WC) and WHR also decreased in all of the groups after 3 months of treatment. However, a greater reduction in $\mathrm{WC}$ was observed in the BBR group $(88.38 \pm 5.84$ vs $80.22 \pm 5.17)$ than in

Table 1 Clinical characteristics and metabolic parameters of all subjects before and after treatment.

\begin{tabular}{|c|c|c|c|c|c|c|}
\hline \multirow[b]{2}{*}{ Parameters } & \multicolumn{2}{|c|}{$\begin{array}{l}\mathbf{B B R}+\mathbf{C A}(n=31 ; \\
\text { age }=25.74 \pm 2.66)\end{array}$} & \multicolumn{2}{|c|}{$\begin{array}{l}\text { MET + CA }(n=30 ; \\
\text { age }=26.03 \pm 2.82)\end{array}$} & \multicolumn{2}{|c|}{$\begin{array}{c}\mathbf{P L}+\mathbf{C A}(n=28 ; \\
\text { age }=26.75 \pm 2.62)\end{array}$} \\
\hline & Pre & Post & Pre & Post & Pre & Post \\
\hline Weight (kg) & $65.11 \pm 4.06$ & $60.06 \pm 3.61$ & $62.98 \pm 5.21$ & $58.58 \pm 4.66$ & $65.13 \pm 5.10$ & $60.16 \pm 3.95$ \\
\hline WC $(\mathrm{cm})$ & $88.38 \pm 5.84$ & $80.22 \pm 5.17$ & $89.12 \pm 7.06$ & $85.03 \pm 6.53^{\|}$ & $85.23 \pm 6.18$ & $80.75 \pm 5.28^{\text {व }}$ \\
\hline BMI $\left(\mathrm{kg} / \mathrm{m}^{2}\right)$ & $25.57 \pm 1.60$ & $23.64 \pm 1.45$ & $24.74 \pm 1.85$ & $23.03 \pm 1.72$ & $24.91 \pm 1.66$ & $23.01 \pm 1.27$ \\
\hline FPG (mmol/l) & $4.97 \pm 0.67$ & $4.36 \pm 0.53^{\S}$ & $5.10 \pm 0.69$ & $4.61 \pm 0.55$ & $5.07 \pm 0.67$ & $4.63 \pm 0.73$ \\
\hline FIN (mU/l) & $19.77 \pm 5.80$ & $13.07 \pm 2.45^{\S}$ & $21.94 \pm 12.43$ & $13.81 \pm 4.92^{\|}$ & $21.04 \pm 10.71$ & $17.80 \pm 4.60$ \\
\hline OGTT (nmol/l) & $7.82 \pm 0.58$ & $6.93 \pm 0.93$ & $7.76 \pm 0.46$ & $7.06 \pm 0.92$ & $7.71 \pm 0.42$ & $7.53 \pm 0.53$ \\
\hline HOMA-IR & $4.38 \pm 1.49$ & $2.55 \pm 0.65^{\S}$ & $4.92 \pm 2.61$ & $2.82 \pm 1.02^{\|}$ & $4.78 \pm 2.57$ & $3.76 \pm 0.92$ \\
\hline FGIR & $4.81 \pm 1.19$ & $6.19 \pm 1.25^{\S}$ & $4.85 \pm 1.50$ & $6.60 \pm 2.10^{\|}$ & $4.82 \pm 1.23$ & $5.09 \pm 1.21$ \\
\hline $\mathrm{AUC}_{\mathrm{INS}}$ & $231.55 \pm 61.72$ & $121.66 \pm 14.72^{\S}$ & $258.84 \pm 90.59$ & $122.43 \pm 11.80^{\|}$ & $238.69 \pm 59.97$ & $134.74 \pm 16.39$ \\
\hline $\mathrm{TC}(\mathrm{mmol} / \mathrm{l})$ & $5.85 \pm 0.39$ & $4.65 \pm 0.52^{\S}$ & $5.70 \pm 0.51$ & $5.01 \pm 0.42$ & $5.71 \pm 0.47$ & $5.03 \pm 0.39^{\ddagger}$ \\
\hline TG (mmol/l) & $2.35 \pm 0.27$ & $1.95 \pm 0.24^{*}$ & $2.26 \pm 0.25$ & $2.09 \pm 0.22$ & $2.30 \pm 0.24$ & $2.10 \pm 0.23^{\ddagger}$ \\
\hline HDLC (mmol/li) & $1.11 \pm 0.12$ & $1.24 \pm 0.09^{\S}$ & $1.13 \pm 0.13$ & $1.19 \pm 0.10^{\dagger}$ & $1.11 \pm 0.12$ & $1.13 \pm 0.11^{\ddagger}$ \\
\hline TT $(\mathrm{nmol} / \mathrm{l})$ & $1.89 \pm 0.14$ & $1.47 \pm 0.22^{*}$ & $1.88 \pm 0.13$ & $1.53 \pm 0.20$ & $1.85 \pm 0.12$ & $1.59 \pm 0.17$ \\
\hline SHBG $(\mathrm{nmol} / \mathrm{l})$ & $25.37 \pm 4.31$ & $58.70 \pm 11.03^{\S}$ & $24.20 \pm 3.52$ & $51.98 \pm 9.66^{\| \prime}$ & $24.19 \pm 4.01$ & $45.01 \pm 8.32^{\top}$ \\
\hline FAI (\%) & $7.69 \pm 1.55$ & $2.59 \pm 1.12^{\S}$ & $7.92 \pm 1.22$ & $3.16 \pm 0.98^{\dagger}$ & $7.81 \pm 1.15$ & $3.77 \pm 1.38$ \\
\hline
\end{tabular}


the MET group $(89.12 \pm 7.06$ vs $85.03 \pm 6.53$; $P=0.001)$. WHR decreased from $0.89 \pm 0.03$ to $0.82 \pm 0.04$ in the BBR group, a significantly greater reduction than that observed in the MET $(0.9 \pm 0.04$ vs $0.87 \pm 0.03 ; P<0.001)$ and the placebo groups $(0.89$ \pm 0.05 vs $0.87 \pm 0.44 ; P<0.001$ ) (Table 1 ).

\section{Carbohydrate metabolic parameter of the studied subjects}

After 3 months of treatment, the placebo group, FIN, HOMA-IR, and $\mathrm{AUC}_{\mathrm{INS}}$ showed a significant reduction compared with the baseline (Table 1). Other markers of IR, such as FGIR and FPG, remained unchanged; however, compared with the subjects who received placebo, subjects receiving BBR or MET had a significant improvement in insulin sensitivity. FPG decreased from $4.97 \pm 0.67$ to $4.36 \pm 0.53$ with BBR $(P=0.005)$. FIN significantly decreased after treatment with BBR $(19.77 \pm 5.80$ vs $13.07 \pm 2.45)$ or MET (21.94 \pm 12.43 vs $13.81 \pm 4.92)$. Moreover, a significant decrease in HOMA-IR and $\mathrm{AUC}_{\mathrm{INS}}$ was also detected in the BBR and MET groups. Conversely, FGIR was significantly increased after treatment with BBR $(4.81 \pm 1.19$ vs $6.19 \pm 1.25 ; P=0.004)$ or $\operatorname{MET}(4.85 \pm 1.50$ vs $6.60 \pm 2.10 ; P=0.001)$. However, no significant variation in FPG, FIN, FGIR, HOMA-IR, and $\mathrm{AUC}_{\mathrm{INS}}$ was noted between the BBR and MET groups (Table 1).

\section{Lipid profile parameters of the studied subjects}

After 3 months of treatment, reductions in TC and TG were observed in all of the groups. In the placebo group, the level of LDLC showed a decreasing trend but was not statistically significant, and the level of HDLC remained unchanged. The BBR group demonstrated a significant decrease in TG from $2.35 \pm 0.27$ to $1.95 \pm 0.24$, TC from $5.58 \pm 0.39$ to $4.65 \pm 0.52$, and LDLC from $4.22 \pm 0.56$ to $3.62 \pm 0.60$. Compared with the MET group, the differences of TG, TC, and LDLC were statistically significant $(P=0.034, P=0.012, P=0.016)$. Additionally, BBR therapy caused a significant increase in the plasma level of HDLC from $1.11 \pm 0.12$ to $1.24 \pm 0.09$, whereas MET treatment only increased HDLC from $1.13 \pm 0.13$ to $1.19 \pm 0.10(P=0.039)($ Table 1$)$.

\section{Reproductive hormone levels of the studied subjects}

TT and FAI decreased, whereas SHBG increased significantly in all of the groups after 3 months of treatment. The level of SHBG increased significantly in the BBR group $(25.37 \pm 4.31$ vs $58.70 \pm 11.03)$ compared with the MET $(P=0.008)$ and placebo groups $(P=0.006)$. BBR treatment resulted in a significant decline in TT from $1.89 \pm 0.14$ to $1.47 \pm 0.22$ and a corresponding decline of FAI from $7.69 \pm 1.55$ to
$2.59 \pm 1.12$, which were significantly different from the changes in the placebo group $(P=0.017$, $P=0.0002)$. However, the changes in TT and FAI after BBR treatment were not significantly different from the changes observed following MET treatment $(P=0.15$, $P=0.06$ ) (Table 1).

\section{Discussion}

In recent years, the recommended management strategy for long-term treatment of PCOS includes lifestyle modification, insulin sensitizers, and OC. This comprehensive intervention protocol results in better regularity of menses and fertility potential $(10,29,30)$. In this study, OC and lifestyle modification remained the first-line therapy choice and every subject received CPA in the form of a combined pill during the 3-month treatment period. Furthermore, BBR, MET, and placebo were administered randomly in order to evaluate the effects of BBR in comparison to MET in a group of women with PCOS. However, this study had two limitations: small subgroups were studied and the study population was under the treatment of OC.

Compared with the baseline, significant improvement in biochemical indices of hyperandrogenemia like TT and FAI, were observed in all of the groups after 3 months of treatment. Our data suggest that both BBR and MET have similar effects on reduction of concentrations of androgens, which are in agreement with the results of other studies analyzing effects of MET in $\operatorname{PCOS}(9,31)$.

MET as the most commonly used insulin sensitizer exerts favorable metabolic outcomes in women with PCOS, such as decrease in insulin and androgen concentrations, normalization of menstrual cycles, and improvement ovulation rates $(10,32,33)$. Although, our 3-month treatment period led to decrease in concentrations of insulin and androgens as it was reported elsewhere $(11,12,32,33)$, we could not confirm significant effect of MET on weight change and lipid profile as it was shown by others $(11,12,34)$. Three months may be too short a time to demonstrate the effect of MET on metabolic abnormalities sufficiently. Furthermore, MET is associated with a higher incidence of nausea, vomiting, and other gastrointestinal disturbances.

$\mathrm{BBR}$, as the main constituent of traditional Chinese medicine, displays good potential in the prevention and treatment of metabolic disorders, including weight control, cholesterol reduction, and antilipogenic and hypoglycemic effects (35). Several trials have reported that BBR treatment can not only reduce body weight and the ratio of white adipose tissue to body weight $(7,14)$ but also increase energy expenditure and the consumption of lipid metabolites as the primary energy source in obese animals (36). In our study, body weight and BMI decreased in all three groups after treatment. Compared with the placebo group, subjects treated with 
BBR showed no significant differences in body weight and BMI. Therefore, losing weight may be attributed to lifestyle modifications rather than BBR therapy, which conflicts with previous results that demonstrated BBR's anti-obesity activity $(14,37)$. It is possible that the treatment period did not last long enough for the effect of BBR on body weight to be observed. However, WC and WHR in the BBR group decreased significantly compared with the MET and placebo groups, which supports the notion of BBR-induced adipose tissue redistribution and ameliorated central fat distribution in PCOS, with significantly decreased WC and WHR of the subjects in the absence of weight changes.

BBR has been reported to exert hypoglycemic effect in either animal models or on humans and has a comparable activity to MET as an insulin sensitizer. Moreover, BBR improves IGT and reduces blood glucose without increased insulin release and synthesis (38). A growing body of evidence suggests that BBR improves insulin sensitivity and stimulates glucose uptake via activation of the AMP-activated protein kinase pathway (14, 39-41), which may be the target for BBR-induced regulation of glucose and lipid metabolism. Our study confirmed that administration of BBR (0.5 g t.i.d.) was able to reduce FBG, FIN, HOMA-IR, and $\mathrm{AUC}_{\mathrm{INS}}$ in patients with PCOS, and the outcomes were consistent with previous reports. However, no significant variations in glycemic parameters were noted between the BBR and the MET groups. It could be demonstrated that BBR and MET exerted comparable effects on improving glucose metabolism in PCOS.

In addition to the hypoglycemic action, a beneficial effect of BBR on lipid metabolism was also observed in animals and human subjects $(7,14,15,37,42)$. The mechanism of BBR-moderated lipid metabolism is related to upregulation of LDL receptor in both mRNA and protein in liver (7). In this study, the effect of BBR on lipid metabolic parameters was demonstrated by the significant decrease in serum concentrations of TC, TG, and LDLC and the HDLC increase after 3 months of treatment. Although BBR and MET both improved glycemic parameters, their effects on lipid metabolism were different. Compared with BBR, MET was less effective on the lipid parameters. BBR-induced alleviation of lipid dysregulation surpassed those obtained by MET in this study.

Until now, there have been few reports concerning the effect of BBR use on reproductive hormones. A recent study with dexamethasone-induced IR on theca cells showed an excessive testosterone production and that was effectively antagonized by BBR (43). In our study, level of testosterone and FAI significantly decreased in the group treated with BBR in comparison to the placebo group. Although the mechanism of BBRreducing hyperandrogenism remains to be clarified, it may be partially related to the remarkable effect of BBR on amelioration of IR.
With regard to safety, BBR is not considered toxic at present doses used in clinical situations (37). Recent studies reported that by treatment with BBR $(0.5 \mathrm{~g}$ t.i.d.) none of the subjects were observed to have marked changes in renal and hepatic function. The pharmacokinetics of BBR studied in rats also suggests that blood clearance of BBR is very quick and that its biotransformation in the liver is rapid (44). The major side effects of BBR can result from overdose, including diarrhea, constipation, flatulence, and abdominal pain in rare cases (7). Subjects in this clinical study tolerated BBR well and none had mild gastrointestinal discomfort. No data are available regarding the safety of BBR in long-term use in young women and on its safety in early pregnancy.

In conclusion, we showed that BBR in comparison to MET showed similar metabolic effects presumably on amelioration of insulin sensitivity and reduction of hyperandrogenemia. BBR also appeared to have a greater effect on the changes in body composition and dyslipidemia. However, the underlying mechanisms of its action remain to be clarified in longer term clinical trials that are required to evaluate therapeutic efficacy and safety of BBR in women with PCOS.

\section{Declaration of interest}

The authors declare that there is no conflict of interest that could be perceived as prejudicing the impartiality of the research reported.

\section{Funding}

This research did not receive any specific grant from any funding agency in the public, commercial, or not-for-profit sector.

\section{References}

1 Rotterdam ESHRE/ASRM Sponsored PCOS Consensus Workshop Group. Revised 2003 consensus on diagnostic criteria and longterm health risks related to polycystic ovary syndrome. Fertility and Sterility 200481 19-252.

2 Orio F Jr, Palomba S, Spinelli L, Cascella T, Tauchmanova L, Zullo F, Lombardi G \& Colao A. The cardiovascular risk of young women with polycystic ovary syndrome: an observational, analytical, prospective case-control study. Journal of Clinical Endocrinology and Metabolism 200489 3696-3701. (doi:10.1210/jc.2003032049)

3 Robert A. Wild. Long-term health consequences of PCOS. Human Reproduction Update 20028 231-241. (doi:10.1093/humupd/8. 3.231)

4 Manneras-Holm L, Baghaei F, Holm G, Janson PO, Ohlsson C, Lonn M \& Stener-Victorin E. Coagulation and fibrinolytic disturbances in women with polycystic ovary syndrome. Journal of Clinical Endocrinology and Metabolism 201196 1068-1076. (doi:10.1210/ jc. 2010-2279)

5 Legro RS, Castracane VD \& Kauffman RP. Detecting insulin resistance in polycystic ovary syndrome: purposes and pitfalls. Obstetrical and Gynecological Survey 200459 141-154. (doi:10. 1097/01.OGX.0000109523.25076.E2) 
6 DeUgarte CM, Bartolucci AA \& Azziz R. Prevalence of insulin resistance in the polycystic ovary syndrome using the homeostasis model assessment. Fertility and Sterility 200583 1454-1460. (doi:10.1016/j.fertnstert.2004.11.070)

7 Kong W, Wei J, Abidi P, Lin M, Inaba S, Li C, Wang Y, Wang Z, Si S, Pan H, Wang S, Wu J, Wang Y, Li Z, Liu J \& Jiang JD. Berberine is a novel cholesterol-lowering drug working through a unique mechanism distinct from statins. Nature Medicine 2004 10 1344-1351. (doi:10.1038/nm1135)

8 Eisenhardt S, Schwarzmann N, Henschel V, Germeyer A, Wolff M, Hamann A \& Strowitzki T. Early effects of metformin in women with polycystic ovary syndrome: a prospective randomized, double-blind, placebo-controlled trial. Journal of Clinical Endocrinology and Metabolism 200691 946-952. (doi:10.1210/ jc.2005-1994)

9 Hoeger K, Davidson K, Kochman L, Cherry T, Kopin L \& David SG. The impact of metformin, oral contraceptives, and lifestyle modification on polycystic ovary syndrome in obese adolescent women in two randomized, placebo-controlled clinical trials. Journal of Clinical Endocrinology and Metabolism 200893 4299-4306. (doi:10.1210/jc.2008-0461)

10 Diamanti-Kandarakis E, Paterakis T, Alexandraki K, Piperi C, Aessopos A, Katsikis I, Katsilambros N, Kreatsas G \& Panidis D. Indices of low-grade chronic inflammation in polycystic ovary syndrome and the beneficial effect of metformin. Human Reproduction 200621 1426-1431. (doi:10.1093/humrep/de 1003)

11 Trolle B, Flyvbjerg A, Kesmodel U \& Lauszus FF. Efficacy of metformin in obese and non-obese women with polycystic ovary syndrome: a randomized, double-blinded, placebo-controlled cross-over trial. Human Reproduction 2007 22 2967-2973. (doi:10.1093/humrep/dem271)

12 Harborne LR, Sattar N, Norman JE \& Fleming R. Metformin and weight loss in obese women with polycystic ovary syndrome: comparison of doses. Journal of Clinical Endocrinology and Metabolism 200590 4593-4598. (doi:10.1210/jc.2004-2283)

13 Macut D, Panidis D, Glisic B, Spanos N, Petakov M, Bjekic J, Stanojlovic O, Rousso D, Kourtis A, Bozic I \& Damjanovic S. Lipid and lipoprotein profile in women with polycystic ovary syndrome. Canadian Journal of Physiology and Pharmacology 2008 86 199-204. (doi:10.1139/Y08-014)

14 Lee YS, Kim WS, Kim KH, Yoon MJ, Cho HJ, Shen Y, Ye JM, Lee CH, Oh WK, Kim CT, Hohnen-Behrens C, Gosby A, Kraegen EW, James DE \& Kim JB. Berberine, a natural plant product, activates AMP-activated protein kinase with beneficial metabolic effects in diabetic and insulin-resistant states. Diabetes Care 200655 2256-2264.

15 Yin J, Xing H \& Ye J. Efficacy of berberine in patients with type 2 diabetes mellitus. Metabolism 200857 712-717. (doi:10.1016/ j.metabol.2008.01.013)

16 Gu Y, Shi X, Li X, Hong J, Chen J, Gu W, Lu X, Xu G \& Ning G. Effect of traditional Chinese medicine berberine on type 2 diabetes based on comprehensive metabonomics. Talanta $2010 \mathbf{8 1} 766-772$. (doi:10.1016/j.talanta.2010.01.015)

17 Yin J, Gao Z, Liu D, Liu Z \& Ye J. Berberine improves glucose metabolism through induction of glycolysis. American Journal of Physiology. Endocrinology and Metabolism 2008294 E148-E156. (doi:10.1152/ajpendo.00211.2007)

18 Ascaso JF, Romero P, Real JT, Lorente RI, Martinez-Valls J \& Carmena R. Abdominal obesity, insulin resistance, and metabolic syndrome in a southern European population. European Journal of Internal Medicine 200314 101-106. (doi:10.1016/ S0953-6205(03)00022-0)

19 Pai JK, Pischon T, Ma J, Manson JE, Hankinson SE, Joshipura K, Curhan GC, Rifai N, Cannuscio CC, Stampfer MJ \& Rimm EB. Inflammatory markers and the risk of coronary heart disease in men and women. New England Journal of Medicine 2004351 2599-2610. (doi:10.1056/NEJMoa040967)

20 Hatch R, Rosenfield RL, Kim MH \& Tredway D. Hirsutism: implications, etiology, and management. American Journal of Obstetrics and Gynecology $1981 \mathbf{1 4 0} 815-830$.
21 Laven JS, Imani B, Eijkemans MJ \& Fauser BC. New approach to polycystic ovary syndrome and other forms of anovulatory infertility. Obstetrical and Gynecological Survey 200257 755-767. (doi:10.1097/00006254-200211000-00022)

22 Yue J. Obstetrics and gynecology. People's Medical Publishing House, 6th edn, ch 46, p 418. Peking: Xinhua Book Store, 2004.

23 Balen AH, Laven JS, Tan SL \& Dewailly D. Ultrasound assessment of the polycystic ovary: international consensus definitions. Human Reproduction Update 20039 505-514. (doi:10.1093/ humupd/dmg044)

24 Matthews DR, Hosker JP, Rudenski AS, Naylor BA, Treacher DF \& Turner RC. Homeostasis model assessment: insulin resistance and beta-cell function from fasting plasma glucose and insulin concentrations in man. Diabetologia 198528 412-419. (doi:10. 1007/BF00280883)

25 Legro RS, Finegood D \& Dunaif A. A fasting glucose to insulin ratio is a useful measure of insulin sensitivity in women with polycystic ovary syndrome. Journal of Clinical Endocrinology and Metabolism 199883 2694-2698. (doi:10.1210/jc.83.8.2694)

26 Mathur S, Baker ER, Williamson HO, Derrick FC, Teague KJ \& Fudenberg HH. Clinical significance of sperm antibodies in infertility. Fertility and Sterility 198136 486-495.

27 WHO Technical Report Series, No. 646, 1980 (Second report of the WHO Expert Committee on Diabetes Mellitus).

28 Haffner SM, Valdez RA, Hazuda HP, Mitchell BD, Morales PA \& Stern MP. Prospective analysis of the insulin resistance syndrome (syndrome X). Diabetes $1992 \mathbf{4 1}$ 715-722. (doi:10.2337/ diabetes.41.6.715)

29 Pasquali R, Gambineri A, Cavazza C, Ibarra Gasparini D, Ciampaglia W, Cognigni GE \& Pagotto U. Heterogeneity in the responsiveness to long-term lifestyle intervention and predictability in obese women with polycystic ovary syndrome. European Journal of Endocrinology $2011 \mathbf{1 6 4} 53-60$. (doi:10.1530/EJE-100692)

30 Hoeger KM, Kochman L, Wixom N, Craig K, Miller RK \& Guzick DS. A randomized, 48-week, placebo-controlled trial of intensive lifestyle modification and/or metformin therapy in overweight women with polycystic ovary syndrome: a pilot study. Fertility and Sterility 2004 82 421-429. (doi:10.1016/j.fertnstert. 2004.02.104)

31 Norman RJ, Davies MJ, Lord J \& Moran LJ. The role of lifestyle modification in polycystic ovary syndrome. Trends in Endocrinology and Metabolism 200213 1630-1635.

32 Luque-Ramirez M \& Escobar-Morreale HF. Treatment of polycystic ovary syndrome (PCOS) with metformin ameliorates insulin resistance in parallel with the decrease of serum interleukin-6 concentrations. Hormone and Metabolic Research 201042 815-820. (doi:10.1055/s-0030-1262855)

33 Lord J, Thomas R, Fox B, Acharya U \& Wilkin T. The effect of metformin on fat distribution and the metabolic syndrome in women with polycystic ovary syndrome - a randomised, doubleblind, placebo-controlled trial. British Journal of Obstetrics and Gynaecology 2006113 817-824. (doi:10.1111/j.1471-0528. 2006.00966.x)

34 Kim WS, Lee YS, Cha SH, Jeong HW, Choe SS, Lee MR, Oh GT, Park HS, Lee KU, Lane MD \& Kim JB. Berberine improves lipid dysregulation in obesity by controlling central and peripheral AMPK activity. American Journal of Physiology. Endocrinology and Metabolism $2009 \mathbf{2 9 6}$ E812-E819. (doi:10.1152/ajpendo. 90710.2008)

35 Vuddanda PR, Chakraborty S \& Singh S. Berberine: a potential phytochemical with multispectrum therapeutic activities. Expert Opinion on Investigational Drugs 201019 1297-1307. (doi:10. $1517 / 13543784.2010 .517745)$

36 Chen C, Zhang Y \& Huang C. Berberine inhibits PTP1B activity and mimics insulin action. Biochemical and Biophysical Research Communications 2010397 543-547. (doi:10.1016/j.bbrc.2010. 05.153)

37 Zhang YF, Li XY, Zou DJ, Liu W, Yang JL, Zhu N, Huo L, Wang M, Hong J, Wu PH, Ren GG \& Ning G. Treatment of type 2 diabetes and 
dyslipidemia with the natural plant alkaloid berberine. Journal of Clinical Endocrinology and Metabolism 200893 2559-2565. (doi:10. 1210/jc.2007-2404)

38 Zhou L, Yang Y, Wang X, Liu S, Shang W, Yuan G, Li F, Tang J, Chen $M$ \& Chen J. Berberine stimulates glucose transport through a mechanism distinct from insulin. Metabolism 2007 56 405-412. (doi:10.1016/j.metabol.2006.10.025)

39 Cheng Z, Pang T, Gu M, Gao AH, Xie CM, Li JY, Nan FJ \& Li J. Berberine-stimulated glucose uptake in L6 myotubes involves both AMPK and p38 MAPK. Biochimica et Biophysica Acta 2006 760 1682-1689.

40 Yin J, Hu R, Chen M, Tang J, Li F, Yang Y \& Chen J. Effects of berberine on glucose metabolism in vitro. Metabolism $2002 \mathbf{5 1}$ 1439-1443. (doi:10.1053/meta.2002.34715)

41 Turner N, Li JY, Gosby A, To SW, Cheng Z, Miyoshi H, Taketo MM, Cooney GJ, Kraegen EW, James DE, Hu LH, Li J \& Ye JM. Berberine and its more biologically available derivative, dihydroberberine, inhibit mitochondrial respiratory complex I: a mechanism for the action of berberine to activate AMP-activated protein kinase and improve insulin action. Diabetes $2008 \mathbf{5 7}$ 1414-1418. (doi:10.2337/db07-1552)
42 Brusq JM, Ancellin N, Grondin P, Guillard R, Martin S, Saintillan Y \& Issandou M. Inhibition of lipid synthesis through activation of AMP kinase: an additional mechanism for the hypolipidemic effects of berberine. Journal of Lipid Research 200647 1281-1288. (doi:10.1194/jlr.M600020-JLR200)

43 Zhao L, Han F, Hou L, Baillargeon JP, Kuang H, Wang Y \& Wu X. Berberine reduces insulin resistance induced by dexamethasone in theca cells in vitro. Fertility and Sterility 201195 461-463. (doi:10.1016/j.fertnstert.2010.07.1090)

44 Zuo F, Nakamura N, Akao T \& Hattori M. Pharmacokinetics of berberine and its main metabolites in conventional and pseudo germ-free rats determined by liquid chromatography/ion trap mass spectrometry. Drug Metabolism and Disposition $20063 \mathbf{3 4}$ 2064-2072. (doi:10.1124/dmd.106.011361)

Received 9 May 2011

Revised version received 12 October 2011

Accepted 21 October 2011 